\title{
Fingerprinting of brain disease: Connectome identifiability in cognitive decline and
}

\section{neurodegeneration}

Sara Stampacchia ${ }^{1, *}$, Saina Asadi ${ }^{1}$, Szymon Tomczyk ${ }^{2}$, Federica Ribaldi², Max Scheffler ${ }^{8}$, Karl-Olof Lövblad ${ }^{1,3}$, Michela Pievani ${ }^{4}$, Giovanni B. Frisoni ${ }^{2,5}$, Valentina Garibotto ${ }^{1,6, \dagger}$ \& Enrico Amico $^{1,7,+, *}$

${ }^{1}$ Department of Radiology and Medical Informatics, University of Geneva \& Geneva University Neurocenter, Switzerland

${ }^{2}$ Department of Psychiatry, University of Geneva \& Geneva University Neurocenter, Switzerland

${ }^{3}$ Neurodiagnostic and Neurointerventional Division, Geneva University Hospitals, Switzerland

${ }^{4}$ Lab of Alzheimer's Neuroimaging \& Epidemiology, IRCCS Istituto Centro San Giovanni di Dio Fatebenefratelli, Brescia, Italy

5 Memory Center, Department of Rehabilitation and Geriatrics, Geneva University Hospitals, Switzerland

${ }^{6}$ Division of Nuclear Medicine and Molecular Imaging, Geneva University Hospitals, Geneva, Switzerland

${ }^{7}$ Institute of Bioengineering, Center for Neuroprosthetics, EPFL, Geneva, Switzerland

${ }^{8}$ Division of Radiology, Geneva University Hospitals, Geneva, Switzerland

*corresponding authors: sara.stampacchia@gmail.com, enrico.amico@epfl.ch

${ }^{\dagger}$ The authors contributed equally 


\begin{abstract}
In analogy to the friction ridges of a human finger, the functional connectivity patterns of the human brain can be used to identify a given individual from a population. In other words, functional connectivity patterns constitute a marker of human identity, or a 'brain fingerprint'. Notably, very little is known about whether brain fingerprints are preserved in brain ageing and in the presence of cognitive decline. Using fMRI data of 96 memory clinic subjects, here we show that individuals functional connectivity profiles remain unique even when cognitive impairment occurs. Yet, the patterns of functional connectivity that make the healthy subjects more identifiable change during cognitive decline, suggesting that the brain undergoes functional reconfiguration. Notably, the functional connections that were the most reliable in healthy cohorts disappeared during cognitive decline, leaving room for other stable connections, adapting to the process of neurodegeneration. We believe that these findings could help in moving towards a more personalised medicine and treatment during cognitive decline, and we hope they will set the ground for clinical fingerprinting of brain disease.
\end{abstract}




\section{Introduction}

The explosion of publicly available neuroimaging datasets ${ }^{1-3}$ in the last few years has provided an ideal benchmark for mapping functional and structural connections in the human brain. At the same time, quantitative analysis of connectivity patterns based on network science has become more commonly used to study the brain as a network ${ }^{4,5}$, giving rise to the scientific field of Brain Connectomics ${ }^{6}$. Seminal work in this research area ${ }^{7,8}$ has paved the way to the new promising avenue of detecting individual differences through brain connectivity features. These studies showed that an individual's functional connectivity patterns estimated from functional resonance magnetic imaging (fMRI) data, also known as functional connectomes (FCs), can constitute a marker of human uniqueness, as they can be used to identify a given individual in a set of functional connectivity profiles from a population ${ }^{7}$. Given the analogy to well-known properties of the papillary ridges of the human finger, the field has taken the name of 'brain fingerprinting' and, since then, the extraction of "fingerprints" from human brain connectivity data has become a new frontier in neuroscience.

The excitement produced by the discovery that brain fingerprints can be extracted from matrices summarising human brain activity, either during rest or when performing a task, is unsurprising, for several reasons. Firstly, it confirms that studying the brain as a network can provide useful tools to get insights into the individual features that distinguish our brains one from the other; and second, it has been shown that brain fingerprints might relate to behavioural and demographic scores ${ }^{9,10}$. Accordingly, efforts have been made to implement ways of maximising and denoising fingerprints from brain data ${ }^{11-13}$. These findings incentivized human neuroimaging studies to advance from inferences at the population level to the single-subject level, and allowed the field to move towards individualised prediction of cognition and behaviour from brain connectomes ${ }^{14-17}$. The next natural step is to explore whether this property of the human brain is maintained during disease. Despite promising findings towards this direction ${ }^{18,19}$, it is to date unclear to what extent FCfingerprints could be used for mapping disease from human brain data.

Dementia and cognitive decline are the final consequence of a series of brain events. In the case of Alzheimer's Disease (AD) - the most common cause of cognitive decline - these entail the accumulation of toxic proteins between and within neurons (e.g., $\beta$-amyloid and/or hyperphosphorylated tau, respectively) concomitant neuronal death and, ultimately, damage to wider structural and functional networks ${ }^{20}$. From this perspective, AD is considered a disconnection syndrome' ${ }^{21}$ and, over the years, a considerable amount of literature has focussed on connectivity alterations in $\mathrm{AD}^{22-24}$.

Cognitive decline due to $\mathrm{AD}$ is accompanied by loss of functional connectivity between regions and loss of network organisation ${ }^{25,26}$. Loss of connectivity however is often accompanied by hyperconnectivity in other brain regions/networks ${ }^{27-30}$, and phases of hyperconnectivity have been described just before the start of the cognitive symptoms ${ }^{31-34}$. Another connectivity feature of cognitive impairment is a change in $h u b$ regions ${ }^{35,36}$. Hubs are regions that mediate a high proportion 
of functional connections and that are thought to be crucial for the integration of information from domain-specific systems in the healthy brain ${ }^{4,37}$. Interestingly, the hubs often correspond to the epicentres of $\beta$-amyloid accumulation ${ }^{35,36,38}$, and some suggest that this susceptibility to pathophysiology could be due to the high metabolic needs of these regions ${ }^{36}$. In line with this, individuals with $\mathrm{AD}$ show loss of nodal centrality in several $h u b$ regions, for example in the medial temporal lobe, posterior portions of the default mode network (DMN), and occipital regions ${ }^{39}$. In addition, the connectivity strength of tau epicentres is strongly predictive of the topography of tau accumulation, suggesting that tau could spread through functional connections ${ }^{40-42}$. Finally, graph theory studies have shown that the global network organisation changes in $\mathrm{AD}$ and becomes characterised by shorter path lengths between connected regions (randomness) and an increased number of hubs (i.e., regularity ${ }^{28}$ ). In sum, the literature suggests that the brain undergoes loss and reorganisation of functional connectivity during pathological ageing, and that the changes are closely linked to the underlying $\beta$-amyloid and tau pathophysiology.

However and despite the considerable amount of literature on the topic, there is not an agreement on the functional connectivity signatures of the various stages and etiologies of cognitive decline ${ }^{23}$, and this has hindered its use as a clinical biomarker ${ }^{32,43}$. This is partly due to the intrinsic properties of resting-state fMRI-connectivity, a technique that is greatly influenced by factors affecting the signal processing (heterogeneity in acquisition parameters, scanners characteristics, motion ${ }^{44}$ ) by differences in the analytical approaches chosen ${ }^{32}$, and also by the fact that existing studies focused on group averages, overlooking heterogeneity among individuals. The existence of inter-subjects variability in terms of cognitive phenotypes - but also biological features - is wellrecognized in clinical practice and, as discussed above, there is evidence that this cognitive variability can be explained by changes in functional organisation. Addressing the deep research question of fingerprinting during brain disease could therefore open the door to individual characterization of cognitive decline from functional connectivity data and open the way to its use in clinical settings. Investigating fingerprints of cognitive decline is tightly connected to the concept of "precision medicine" ${ }^{45}$, since it might provide insights on the individual trajectories of pathological brain ageing and/or personalised treatment during cognitive decline, advancing medicine in its quest for individualised biomarkers of neurodegeneration ${ }^{46}$.

In this work, we investigated brain connectivity fingerprints using fMRI data collected from 96 individuals at different stages of cognitive decline. We started by estimating functional connectome fingerprints of an heterogeneous cohort of Cognitively Unimpaired (CU), Mild Cognitive Impairment (MCI) and dementia, during the first and second half of the fMRI session. We found that whole-brain functional connectivity patterns remained reliable across healthy and pathological brain ageing; in other words, it was possible to correctly identify a patient solely based on its functional connectome. Yet, significant differences in the spatial organisation of the brain fingerprint were observed during cognitive decline. Notably, the functional connections that were the most reliable in 
bioRxiv preprint doi: https://doi.org/10.1101/2022.02 .04.479112; this version posted February 8, 2022. The copyright holder for this preprint (which was not certified by peer review) is the author/funder, who has granted bioRxiv a license to display the preprint in perpetuity. It is made available under aCC-BY-NC-ND 4.0 International license.

healthy cohorts disappeared during cognitive decline, leaving room for other stable connections, adapting to the process of neurodegeneration. We hope that the findings will help in setting the grounds for clinical fingerprinting in fMRI, and in improving our understanding of the link between unique brain connectivity features and individualised biomarkers of neurodegeneration.

\section{$\underline{\text { Results }}$}

We investigated brain fingerprints of neurodegeneration in a cohort of $\mathrm{N}=96$ subjects at different stages of cognitive decline: unimpaired (CU), with mild cognitive Impairment (MCI), and dementia. The approach can be summarised in three steps: (1) We first estimated the functional connectomes (FC) of each subject during the first 100 and second 100 volumes of fMRI acquisitions respectively, (cf. Fig. 1 and see Methods for details). (2) We then estimated the degree of brain identification or "brain fingerprint" at the whole-brain level, through a mathematical object called Identifiability matrix ${ }^{12}$ (cf. Fig 1).

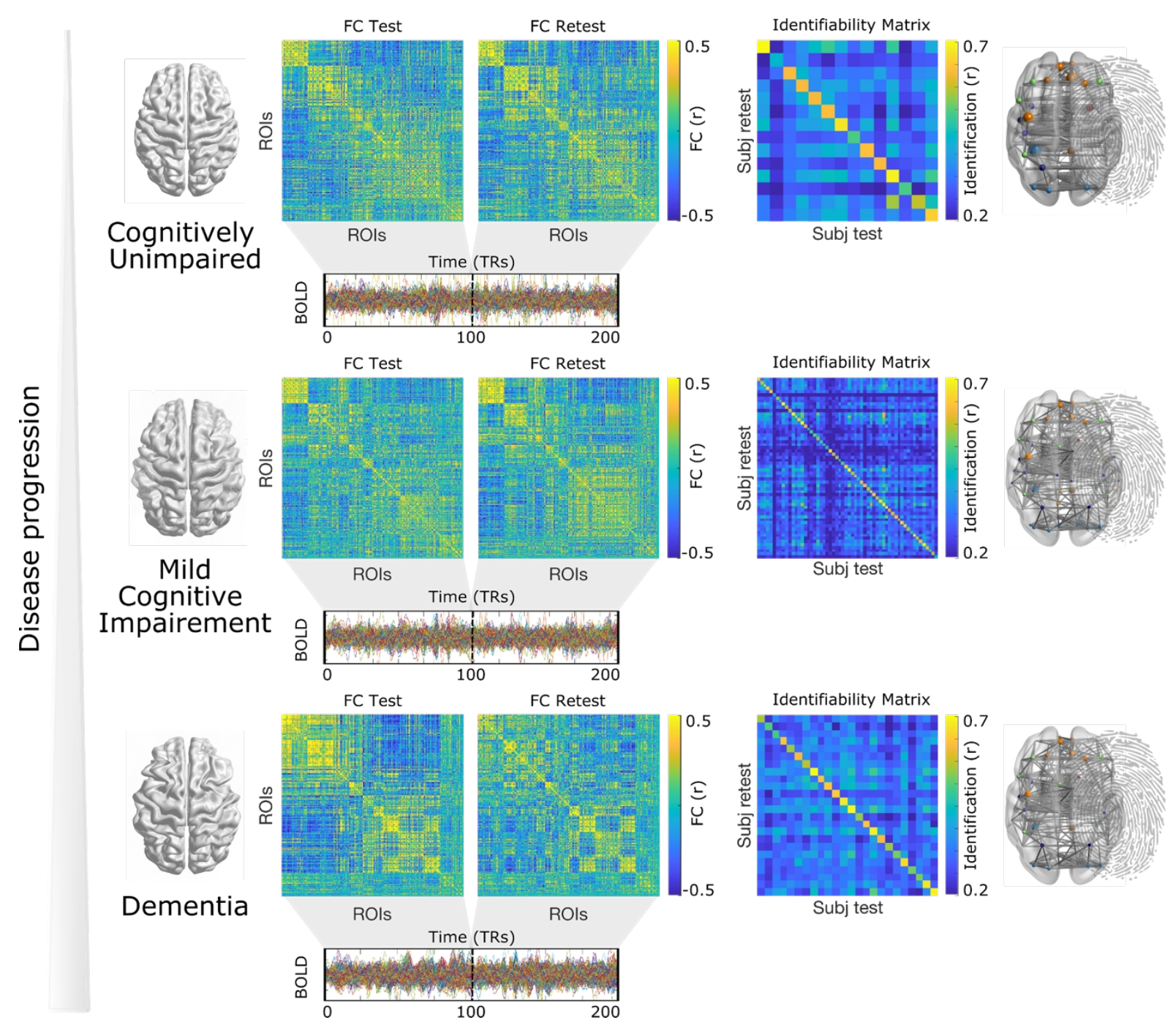


Figure 1. Exploring FC fingerprints of neurodegeneration, schematics of the approach. We estimated the FCfingerprint in Cognitively Unimpaired, Mild Cognitive Impairment and dementia patients. Fingerprint was estimated as the degree of similarity between Functional Connectivity at test (FC Test, first 100 volumes) vs. retest (FC Retest, second 100 volumes) and summarised in a mathematical object called Identifiability Matrix ${ }^{12}$.

The identifiability matrix provides two useful metrics for brain fingerprinting: the degree of similarity of each subject with themselves (ISelf) as opposed to others (IOthers), and the degree of fingerprint, conceptualised as the extent to which subjects were more similar to themselves than others (IDiff, see Methods). We further explored the spatial specificity of brain fingerprints by estimating the degree of distinctiveness of each FC-edge at the individual level, using intraclass correlation (ICC, see Methods). (3) We finally explored the association between brain fingerprints and connectome variability across time, conceptualised here as FC flexibility.

\subsection{Functional Connectivity fingerprints can be detected also in cognitive decline}

At the whole-brain level, the success-rate of the identification procedure was $100 \%$. All cases showed higher similarity with themselves (ISelf), as opposed to others (IOthers), and IDiff was comparably high in the three groups. We also found that test-retest reliability (ISelf) was high in the three groups, with no significant differences across groups [CU: $\mathrm{M}(\mathrm{SD})=0.61(0.07)$; $\mathrm{MCI}$ : $\mathrm{M}(\mathrm{SD})=0.59(0.09)$; Dementia: $\mathrm{M}(\mathrm{SD})=0.60(0.08)$; cf. Fig. 2A]. Note that these ISelf values are equivalent to those found by existing studies in healthy subjects of the Human Connectome Project database ${ }^{47}$. Permutation testing showed that IDiff and Success-rate were different from null distributions at $\mathrm{p}<.001$ in all groups (Fig. 2A). In other words, it proved to be possible to correctly identify an individual in the cohort, with significantly greater accuracy relative to surrogate null models ${ }^{48}$ (see Methods for details), independently from the stage of cognitive decline, and solely based on the patterns of brain activity. Note that the reported identification rates are computed at the whole-brain level, hence giving no information on the functional edges most influential for identification. The ensuing aim was therefore to understand how these results related to the local properties of the individual functional connectomes.

\subsection{The brain undergoes functional reconfiguration during cognitive decline.}

To address this question, we assessed spatial specificity of brain fingerprints using edgewise intra-class correlation ${ }^{12}$ (ICC). Interestingly, we found that the FC-fingerprints of the most identifiable edges followed a U-shaped pattern along neurodegeneration, with an overall reduction in MCI, and an increase in dementia (Fig. 2B and Fig. 2C). In addition, the spatial configuration of the edges with the highest fingerprint changed during cognitive decline (Fig. 2B and Fig. 2D).

In order to further explore the patterns of functional reconfiguration that emerged from the ICC matrices, we analysed the changes in the identification rate (in terms of IDiff) when iteratively selecting the edges from highest to lowest fingerprint (ICC). Specifically, we first ranked the edges 
bioRxiv preprint doi: https://doi.org/10.1101/2022.02.04.479112; this version posted February 8, 2022. The copyright holder for this preprint (which was not certified by peer review) is the author/funder, who has granted bioRxiv a license to display the preprint in perpetuity. It is made available under aCC-BY-NC-ND 4.0 International license.

with the highest ICC in the healthy cohort, and we then calculated IDiff in CU and in the two clinical groups. IDiff was computed on subsets of the first 100, 200, 300, ... ranked FC edges in steps of 100, eventually including all the FC edges ( 40000). As reported in Fig. 2D, this analysis showed that the edges with the highest fingerprint in $\mathrm{CU}$ are not the ones contributing to fingerprinting patients in the two clinical groups. The IDiff scores for MCI and dementia were consistently lower (and stationary) in the top CU edges. Of note, there was a slight decrease in IDiff in the MCI and dementia curve around 1200 edges (Fig. 2D). In conclusion, the edges that ranked as top in identifying the healthy cohort, were not as prominent across pathological brain ageing.

CU

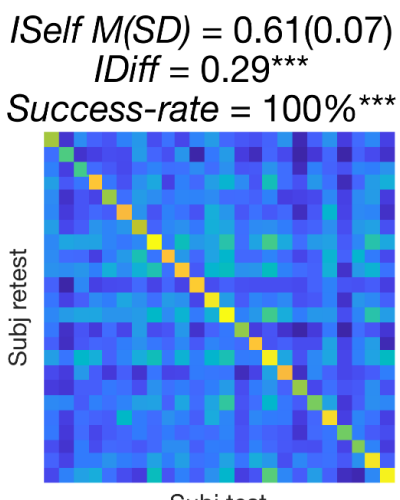

A)

B)

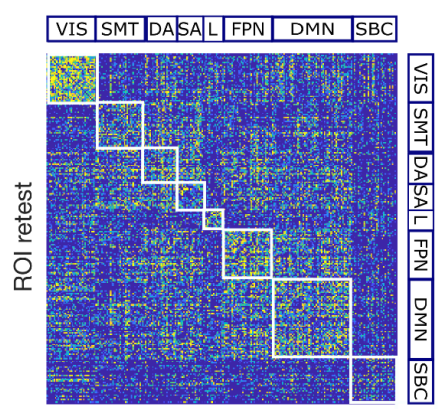

$\mathrm{ROI}$ test

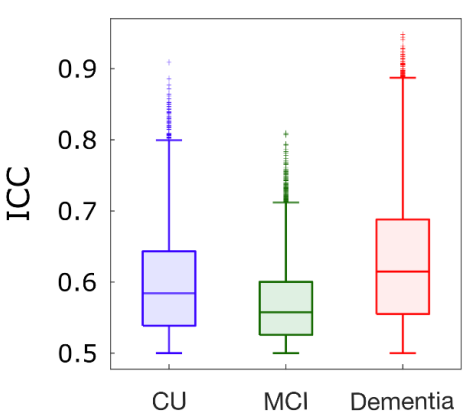

$\mathrm{MCl}$

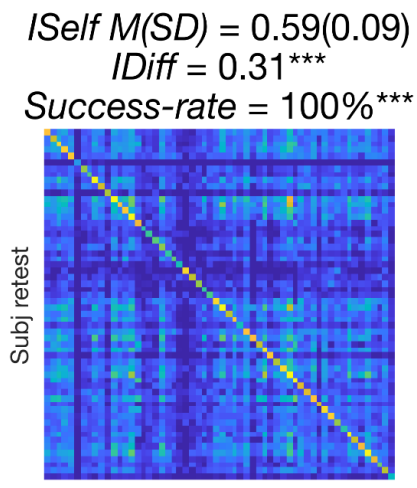

Subj test

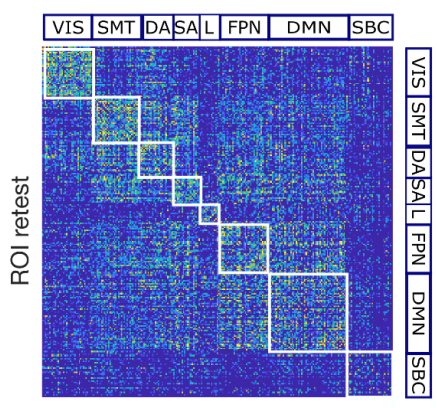

ROI test

\section{Dementia}
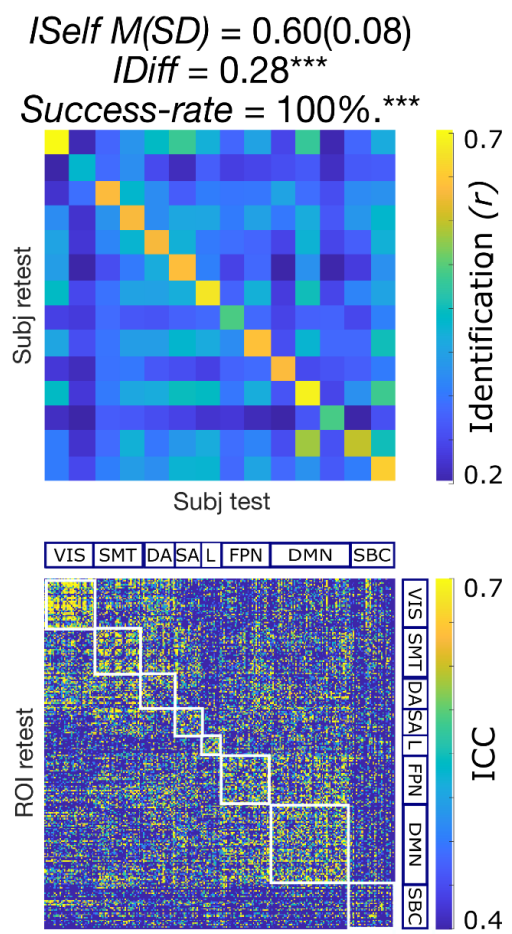

ROI test

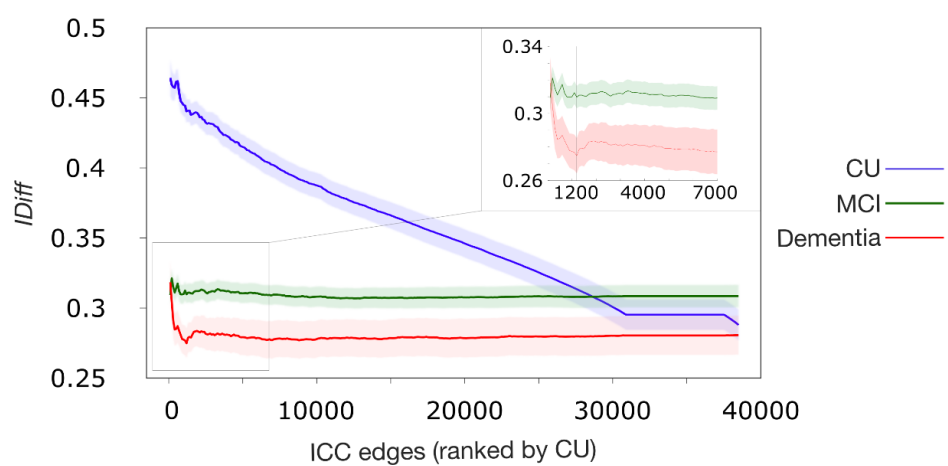

Figure 2. Functional Connectivity fingerprints during cognitive decline. A) Identifiability matrices show within- (ISelf) and between-subjects (IOthers) test-retest reliability as Pearson correlation coefficient in CU, MCI and Dementia. Individuals' ISelf and IOthers are displayed, respectively, in the diagonal and off-diagonal elements of the matrix. The average ISelf, IDiff and Success-rate were similar in the three groups and IDiff and Success-rate differed at $\mathrm{p}<.001$ from random distributions. B) Spatial specificity matrices of FC-fingerprints for each group as measured using edge-wise intraclass correlations (ICC). We display edges with ICC $\geq 0.4$, which is considered good concordance for neuroimaging data 


\begin{abstract}
${ }^{49,50} . \mathrm{VIS}=$ visual network; SMT $=$ somatomotor network; DA = dorsal-attention network; $\mathrm{SA}=$ salience network; $\mathrm{L}=$ limbic network; FPN = fronto-parietal network; DMN = default-mode network; SBC = subcortical regions. C) Boxplots show the U-shaped pattern of the most identifiable edges across cognitive decline, already visible in the ICC matrices. D) Group-level whole-brain estimate of fingerprint (IDiff, see methods below), across subsets of the top 100, 5000,10000, .. 40000 edges with highest edgewise fingerprints (ICC) in Cognitively Unimpaired. The IDiff curves for CU, Mild Cognitive Impairment and dementia for those edges are shown, according to the highest to lowest ICC values in CU. Note that edges with the highest fingerprint in $\mathrm{CU}$ do not correspond to those contributing to fingerprints in patients.
\end{abstract}

We then explored the pattern of functional reconfiguration as expressed by the ICC nodal strength of each brain region. When looking at the fingerprinting hubs in MCI and dementia with respect to $\mathrm{CU}$, we found that individual identification relates to cognitive decline in two fundamental ways (cf. Fig. 3). (i) Regions that are hubs of fingerprinting in CU (i.e., the top three regions with highest ICC) dropped in their prominence in MCI and dementia, specifically: the left inferior frontal gyrus (IFG; percentage difference in nodal strength: $-17.8 \%$ in MCI and $-29.70 \%$ in dementia), the left angular gyrus (AG; $-17.70 \%$ in $\mathrm{MCI}$ and $27.90 \%$ in dementia) and the left posterior middle temporal gyrus (pMTG; $-20.10 \%$ in MCI and $-26.60 \%$ in dementia). Moreover, during dementia the reduction of fingerprints was spread out wider and included neighbouring regions (note in Fig. 3 the spread of blue around AG and pMTG). Finally, there was a notable drop in fingerprinting also in other regions, e.g. see the bilateral posterior cingulate cortex ( $\mathrm{pCC},-27.90 \%)$, right primary visual cortex ($19.20 \%)$, left paracingulate gyrus $(-18.70 \%)$, right superior frontal gyrus $(-16.90 \%)$ in MCI; left inferior temporal gyrus (ITG, $-39.40 \%$ ), left lingual gyrus (-34.30\%), left planum temporale ($33.50 \%$ ), and right anterior cingulate cortex $(-27.20 \%)$ in dementia. (ii) Areas that were not fingerprinting hubs in the healthy, increased their identifiability in MCI and dementia, i.e., the right ventral anterior temporal lobe (vATL, 39\% in MCI and 51.20\% in dementia), the inferior primary motor area (iPMA, $40.20 \%$ in MCI and $44.30 \%$ in dementia) and right posterior superior temporal gyrus (49\% in MCI and 39.6\% in Dementia), the left superior primary motor area (sPMA) in dementia (37.70\%) and bilateral precuneus (PCUN) in MCI (42\%). Table 1 in the Supplementary Materials provides an overview of $\%$ difference in nodal strength. Together with the previous results, this corroborates the finding that during disease there is a reconfiguration of the functional connectivity patterns contributing to the brain fingerprint of an individual.

Finally, we assessed the degree to which edgewise-FC represented a fingerprint of cognitive decline. In order to do so, we used ICC again, this time to quantify the degree to which each FC edge was indicative of the similarity between subjects of the same group (CU, MCI, dementia separately) as well as the degree to which it could distinguish among subjects belonging to the different groups. We found that ICC was very low (ICC $\max =0.03$; for full results cf. Supplementary Materials), confirming what emerged from the inconsistent results in the literature, i.e., that distinguishing among groups using FC data is a difficult task, most likely because of the inter-subjects variability. 
CU

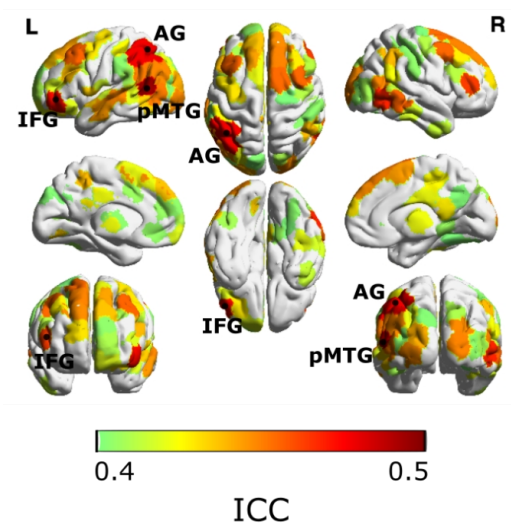

$\mathrm{MCl}$

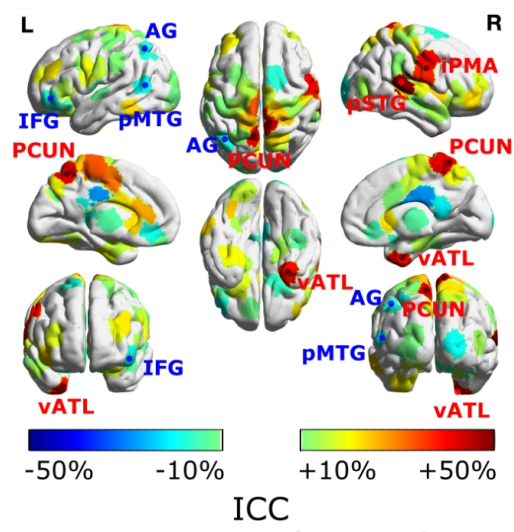

(\% distance from CU)
Dementia

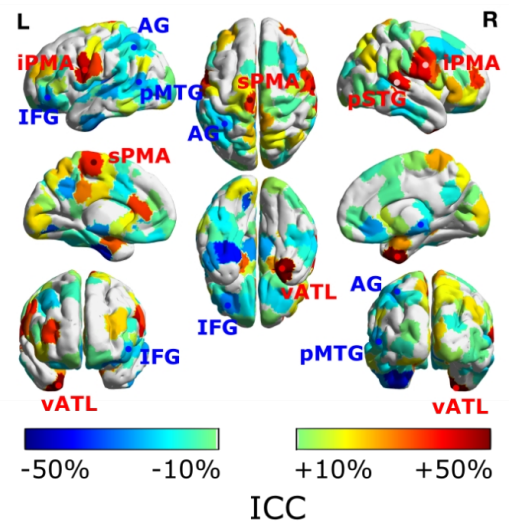

(\% distance from $\mathrm{CU}$ )

Figure 3. The functional reconfiguration of fingerprints during cognitive decline. Brain renders show the most reliable (ICC $\geq 0.4$ ) nodes (or areas) in CU (left). We labelled the top 3 nodes with the highest ICC in CU, namely: left inferior frontal gyrus (IFG), left angular gyrus (AG), left posterior middle temporal gyrus (pMTG). For MCI (middle) and dementia (right) we display percentage difference in nodal strength using CU as reference. Regions labelled in blue are those with the highest fingerprint in $\mathrm{CU}$. Note that these regions are amongst those with the biggest reduction in MCI and dementia. The top regions with bigger reduction in ICC were: for MCI, left and right posterior cingulate cortex (pCC, $-27.9 \%$ ), left posterior middle temporal gyrus (pMTG, -20.1\%), right primary visual cortex (V1, -19.2\%), paracingulate gyrus (-18.7\%), left inferior frontal gyrus (IFG, -17.8\%), left angular gyrus (AG, -17.7\%), right superior frontal gyrus (-16.9\%); for dementia, left inferior temporal gyrus (ITG, $-39.4 \%)$, left lingual gyrus $(-34.3 \%)$, left planum temporale $(-33.5 \%)$, left inferior frontal gyrus (IFG, -29.7\%), left angular gyrus (AG, -27.9\%), right anterior cingulate cortex (aCC, -27.2\%), left posterior middle temporal Gyrus (pMTG, -26.6\%). Regions labelled in red are the top 4 regions with the bigger increase in ICC; for MCI: right posterior superior temporal gyrus (pSTG, 49\%), left and right precuneus (PCUN, $+42 \%$ ), right inferior primary motor area (iPMA, $+40.2 \%$ ), right ventral portion of the anterior temporal lobe (vATL, $+39 \%)$; for dementia: right ventral portion of the anterior temporal lobe $(\mathbf{v A T L},+51.2 \%)$, left and right inferior primary motor area (iPMA, $+44.3 \%)$, right posterior superior temporal gyrus (pSTG, $+39.6 \%$ ), and left superior primary motor area (sPMA, $+37.7 \%$ ).

\subsection{Factors influencing fingerprint reconfiguration}

We investigated potential causes of the fingerprint reconfiguration by exploring whether fingerprints were associated with functional connectome variability across time, here conceptualised as dFC Flexibility (see Methods for details). First, we found that when looking at dFC Flexibility, the U-shaped pattern across cognitive decline re-appeared (Fig. 4A). The most flexible links in CU were between functional networks, specifically between DMN and FPN, and DMN and SAL, SMT and VIS, as well as between VIS and DAN and SMT. This pattern dropped in intensity when transitioning into the MCI condition. Notably, dFC Flexibility was scattered around in dementia, and patterns became less recognizable (Fig. 4A). The regions exhibiting the highest flexibility with the rest of the brain in CU were within the DMN and FPN and corresponded, or were adjacent to those with high fingerprints (cf. Fig. 4B and Fig. 2C). In addition, it can be noted that while the regions exhibiting the highest drop in flexibility in MCI were within DMN and FPN, in dementia networks were no more 
bioRxiv preprint doi: https://doi.org/10.1101/2022.02.04.479112; this version posted February 8, 2022. The copyright holder for this preprint (which was not certified by peer review) is the author/funder, who has granted bioRxiv a license to display the preprint in perpetuity. It is made available under aCC-BY-NC-ND 4.0 International license.

recognizable and drops and increases in dFC Flexibility were limited to specific areas. For example, there were increases in flexibility in the left medial and lateral temporal lobe, as well as in the right insula, orbitofrontal cortex and ventral portion of the ATL.

In order to assess the degree of overlap between fingerprints and flexibility, we assessed their association by correlating their group-averaged edge-wise value (Fig. 4C). We found that although significantly correlated in the three groups $(\mathrm{p}<.0001)$, flexibility only partially explained our findings on brain fingerprints (low correlation coefficient), showing that these metrics represent different features of the functional transition from normal to pathological brain ageing.

Finally, we checked possible associations between edge-wise fingerprint (i.e., ICC matrices) and grey matter atrophy, quantified group-wise as structural covariance matrices (see Methods for details). We found poor correlations between these two metrics across groups (see Supplementary Figure 2).

CU

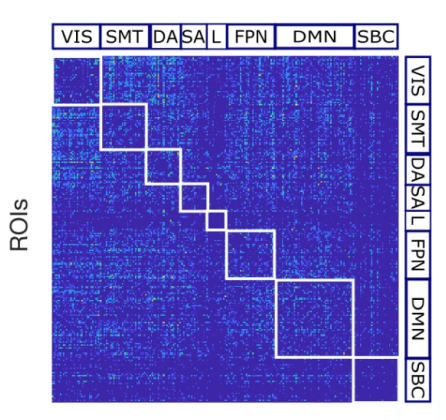

A)

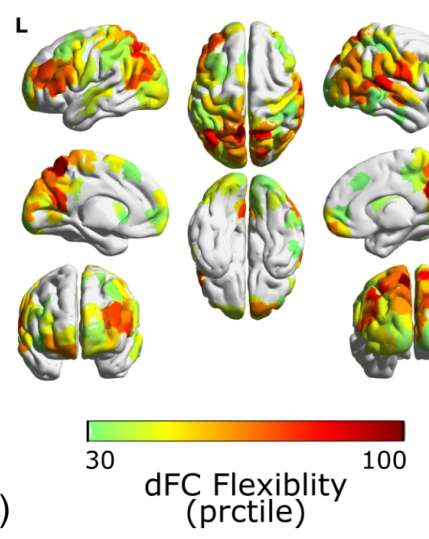

B)

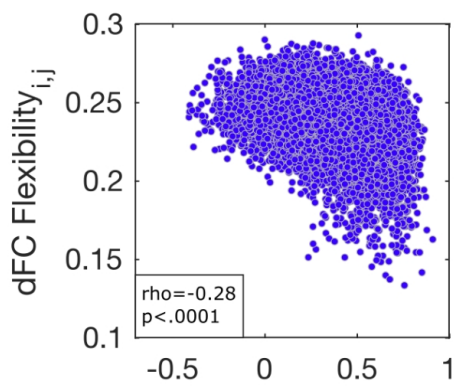

C)

Fingerprint $_{\mathrm{i}, \mathrm{j}}(\mathrm{ICC})$
$\mathrm{MCI}$

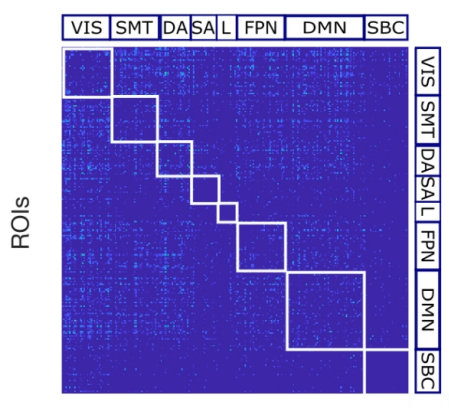

ROls
Dementia

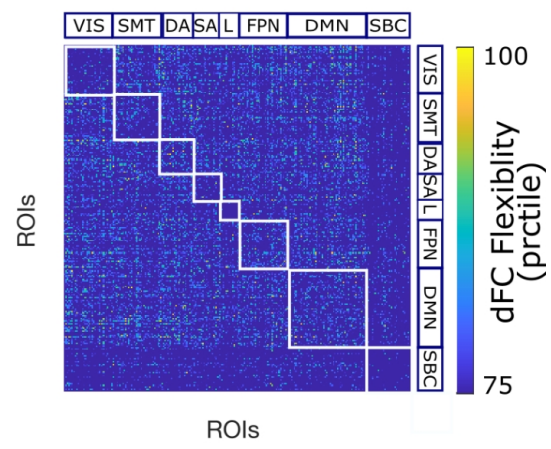

ROIs
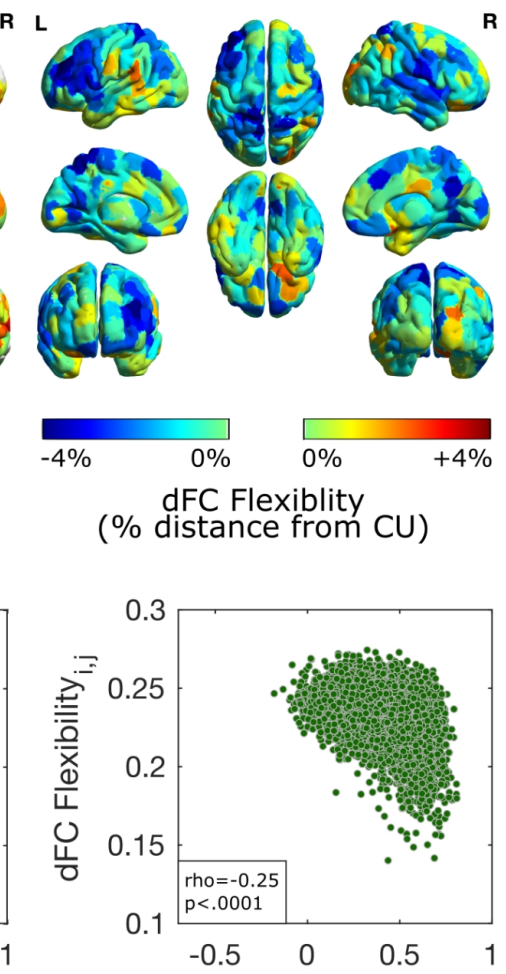

Fingerprint $_{\mathrm{i}, \mathrm{j}}(\mathrm{ICC})$
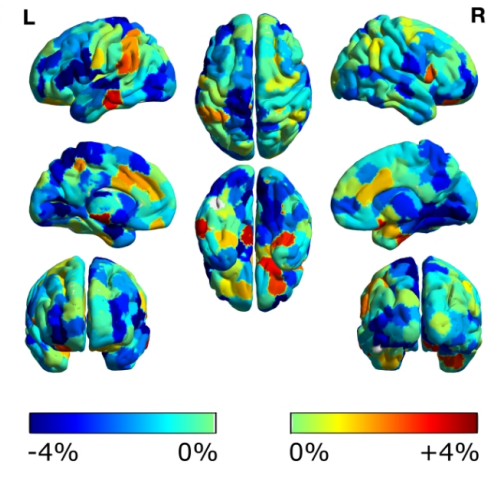

dFC Flexiblity

(\% distance from CU)

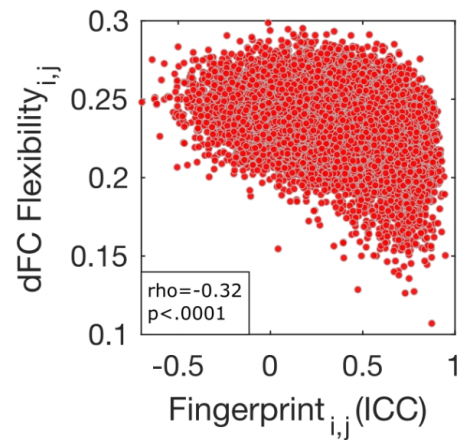


Figure 4. Flexibility and fingerprints: two sides of the same coin. A) Edge-wise connectome flexibility in CU, MCI and dementia is displayed. Edges with high flexibility are displayed $\left(>75^{\text {th }}\right.$ percentile). B) Brain renders show the flexible nodes (or areas) in CU (left) and the percentage difference using CU as reference in MCI (middle) and Dementia (right). In the latter, red areas are those with higher flexibility with respect to $\mathrm{CU}$, while areas in blue reflect a decrease in flexibility. C) Correlation between group average edge-wise fingerprint (ICC) and flexibility. We show that fingerprint and Flexibility (i.e., signal variability across time) are associated yet distinguishable, as shown by low correlation coefficients.

\section{$\underline{\text { Discussion }}$}

In this work, we propose a comprehensive exploration of human brain fingerprints during normal and pathological brain ageing. We evaluated the identification properties of functional connectomes at different stages of cognitive decline using fMRI data, and found that individuals can still be identified during MCI and dementia (Fig. 2A). That is, the uniqueness of the functional connectivity of each individual allows to correctly identify a patient from her/his cohort of reference, independently from how far the neurodegeneration has advanced. In other words, our findings confirm that functional connectivity constitutes a marker of human identity also during cognitive decline.

It is known that the boundaries between stages of cognitive decline are often fuzzy, especially in the early phases, posing critical challenges to the diagnostic and clinical practises. Diagnostic recommendations are periodically reviewed (and debated), and the clinical phenotypes must be analysed in conjunction with molecular pathology and genetics in order to define a comprehensive diagnostic hypothesis ${ }^{43,51,52}$. However, this process is still very much dependent on the subjective clinical judgement because of the individuals' inter-variability. Here we show that the functional connectome of an individual during cognitive decline carries this individualised information that could be precious for patient-specific and patient-oriented evaluation and treatment during neurodegeneration.

To deepen this first observation, we looked at the functional connections contributing to identification and found that in MCI the fingerprint value of the most contributing edges (as measured by ICC, Fig. 2B and Fig. 2C) is reduced across the whole-brain, whereas in dementia there is an increase, creating a peculiar U-shaped pattern. This is in line with previous work where a similar drop in identification between healthy elderly and amnestic MCI was observed, by using magnetoencephalography-based functional connectomes ${ }^{18}$. The increase in identification in dementia patients, however, was unexpected, and led us to a deeper investigation of the U-shaped behaviour of brain fingerprints across pathological ageing. Notably, we observed that the edges and nodes with higher fingerprints in the healthy are reconfiguring in patients (cf. Fig. 2D and Fig. 3). We identified the regions with the highest drop (cf. regions in blue, Fig. 3) and increase (cf. regions in red, Fig. 3) in fingerprint using healthy subjects as a reference.

Intriguingly, among the regions with the greatest drop we found areas implicated in the flexible retrieval of memory, namely left IFG (lying at the intersection between the DMN and the 
frontoparietal network) and left pMTG (between DMN, salience and dorsal-attention networks). An extensive literature has shown that these two regions are key hubs of semantic cognition ${ }^{53-56}$ (and more specifically of 'semantic control') as they are necessary to flexibly retrieve knowledge to suit a specific task or context (for example, to use a newspaper - canonically known for a different purpose to chase a fly). These areas are not exclusively implicated in semantic memory, but also in the cognitive control of other memory domains ${ }^{57-60}$ (i.e., episodic memory), emotions and social processes ${ }^{61}$, suggesting that they may play a broader role in the cognitive control necessary for the attribution of meaning to stimuli and experiences. Also, AG showed a decrease in fingerprint. This region is a key hub of the DMN ${ }^{62}$ and it is consistently activated in a variety of tasks, including semantic and episodic memory ${ }^{63,64}$, sentence reading ${ }^{65}$, number processing and perception ${ }^{66}$. It has been proposed that this region subserves these processes by integrating multimodal representations and by allowing manipulation of mental representations ${ }^{63-65}$.

Our results show that the FC of left IFG, left pMTG and left AG makes healthy elderly individuals identifiable and unique, and these same regions show a drop in fingerprinting through the course of cognitive decline. In other words, the patterns of connectivity of these regions cease to be variable across subjects and, arguably, the pattern of connectivity of these regions with the rest of the brain might need to be highly variable in order to allow for flexible retrieval of memory. According to the reported findings, this property seems to fade over the course of cognitive decline. If replicated on larger cohorts, these results could help in narrowing down the cognitive processes and areas that could be used as the primary targets of cognitive training and/or brain stimulation in the early phases of decline (on the importance of tailored medicine approaches in brain stimulation in AD see ${ }^{67,68}$ ).

Our results also showed that the FC of other regions seems to make people unique even when their cognition declines. Amongst those, the right vATL, which together with its contralateral correspondent, is thought to act as a 'repository' of amodal conceptual knowledge, as confirmed by the detailed study of the striking disorder of Semantic Dementia, where selective atrophy and hypometabolism of the ATLs is associated with selective degradation of semantic knowledge across verbal and non-verbal modalities ${ }^{69,70}$. In absence of the control of LIFG and pMTG, the activity of the ATLs is thought to give rise to rampant and out-of-context retrieval of semantic information (as seen in Semantic Aphasia ${ }^{56,71}$ ). Our data showed that the connectivity of the right vATL with the rest of the brain was not stable across test and retest in the patients, with a highly variable across subjects. This suggests that this region, controlling spontaneous and automatic semantic cognition, might start exhibiting very unique patterns of connectivity during cognitive decline.

The precuneus and neighbouring primary motor areas also increased their identifiability during cognitive decline. The involvement of the motor cortex in AD has been documented in postmortem histological studies that showed a high load of ß-amyloid, but not tau, in this region ${ }^{72}$ and functional connectivity studies who found hyperactivity in this region ${ }^{73}$. Of note, sensorimotor areas are amongst the latest being affected by AD pathology ${ }^{24}$. We here speculate that the increase of 
identifiability observed might be related to increase in the functional segregation of these sensory motor regions.

When ranking the edges based on how reliable they were in identifying the healthy individuals, we found that these edges did not contribute to the identification in the two clinical groups, but rather showed consistently lower identification scores (Fig. 3B). This is in line with the results reported in Fig.2, and confirms the reconfiguration of fingerprints during pathological brain ageing. Note for instance, in Fig. 3, the "drop" in IDiff for MCI and dementia, when considering the top 1200 most identifiable edges for the healthy group (Fig. 3). Our results show that the FC of these regions is what makes healthy elderly individuals identifiable and unique, and these same regions show a drop in fingerprinting throughout the course of cognitive decline.

What are the factors affecting this change in brain fingerprints? We looked at whether the fingerprint was associated with dynamic variability in functional connectivity (connectome flexibility, see Methods for detail). We found that a similar pattern appears across clinical groups. However, connectome flexibility and fingerprints were weakly correlated (albeit significant at $p<0.05$ ), showing that connectome variability (flexibility) and reliability (fingerprints) are two sides of the same coin: two complementary axes through exploring the functional correlates of neurodegeneration. Hence, even though we report a similar (U-shaped) behaviour of these two metrics across brain ageing, the relationship between the two appears to be non-trivial and deserves further investigation. Similarly, we found no association between fingerprint and level of atrophy. We hypothesise that the factors affecting the reconfiguration of fingerprints during cognitive decline are multifaceted, and might involve multiple other variables. Future studies should explore the extent to which atrophy, tau and amyloid accumulations in patients relates to their changes in the fingerprinting spatial patterns.

This study has some limitations. The impact of the choice of the brain atlas should be further verified. It is known that connectivity measures are highly susceptible to artefacts arising from head motion and respiratory fluctuations ${ }^{74,75}$, and these effects are even more pronounced in pathological conditions. However, the data points with high motion did not seem to be a contributor to the differences in fingerprinting across groups, neither in the percentage of censored volumes, nor in the average FD between the groups ( $p>.513)$ and/or test-retest scans $(\mathrm{p}>.680)$. Nonetheless, future work should analyse in depth the effect of motion at shorter time scales, where these artefacts can particularly dominate. Here we used two halves of the same scanning sessions, hence focusing more on the temporal stability of brain identification within-session. Future studies should explore how fingerprints change across scanning sessions, or even longitudinally during cognitive decline (i.e., over days or months). It would be also interesting to further explore the temporality of brain fingerprints in neurodegeneration ${ }^{47}$.

\section{Conclusions}

The friction ridges of a human finger are nearly unique, difficult to alter, and durable over the lifespan of an individual, making them suitable as long-term markers of human identity. We found 
that brain fingerprints also remain unique across individuals in cognitive health and cognitive decline, therefore constituting another marker of human identity. However and intriguingly, during cognitive decline these features are fundamentally different from those of the cognitively normal, suggesting that the brain undergoes a significant functional reconfiguration of its fingerprint during cognitive decline. We provide preliminary evidence that cognitive decline impacts the individual features of FC of the human brain connectivity, in the hope that these first findings could help in setting the grounds for clinical fingerprinting in fMRI. 


\section{Materials and Methods}

\subsection{Participants and demographics}

$\mathrm{N}=101$ subjects from the Geneva Memory Center (GMC, Geneva University Hospitals, Geneva, Switzerland) cohort having (i) a diagnosis made by a trained neurologist on the basis of clinical and neuropsychological assessment, and (ii) fMRI and structural MRI, were included. Maximum time gap between measures $=365$ days. Five subjects were excluded when motioncensored volumes (see below) were $>25 \%$, leaving a total of $\mathrm{N}=96$ subjects.

These included patients diagnosed as mild cognitive impairment (MCI, N=58) or dementia $(\mathrm{N}=14)$, and cognitively unimpaired $(\mathrm{CU}, \mathrm{N}=24)$. The latters were either individuals with subjective cognitive decline (SCD) and/or healthy volunteers with MMSE $\geq 24$ and CDR $=0$ (cf. Table 1). In line with ${ }^{76}$, SCD was defined based on the following criteria: persons asking for help and consulting to the GMC for self-experience of deterioration in cognitive abilities, without objective cognitive impairment detected through formal neuropsychological testing. MCI was defined based on the following clinical criteria: (i) objective evidence of cognitive impairment, (ii) cognitive concern reported by the patient and/or informant (family or close friend), and (iii) little or no functional impairment in daily living activities ${ }^{51}$. Individuals living with dementia are defined based on the same (i) and (ii) above criteria for MCI but differ from them for the impairment in the activities of daily living ${ }^{77}$. Aetiology was defined based on the French National Alzheimer Database diagnostic model 78. Differences across groups in age, years of education and MMSE were tested using Kruskal-Wallis test. Patients (MCI and Dementia) were on average older [p =.064], significantly less educated [p $=.001]$ and with lower MMSE scores $[p<.001]$. There were no gender differences across groups $\left[\chi^{2}=3.84, \mathrm{p}=.147\right]$. In the majority of MCI and dementia cases, cognitive impairment was due to $\mathrm{AD}$ pathology (54.2\%); the remaining was either non-AD cognitive impairment (13.9\%) or unknown (31.9\%). As expected, AD aetiology was more frequent in the cognitively impaired (MCI and dementia) relative to the unimpaired $\left[\chi^{2}=27.25, \mathrm{p}<.001\right]$. Aetiology was unknown mostly in CUs $\left[\chi^{2}=13.44, p=.001\right]$ and the prevalence of non-AD aetiology did not differ across groups $\left[\chi^{2}=2.46\right.$, $\mathrm{p}=.348]$. 
Table 1. Demographics

\begin{tabular}{lcccc} 
& CU & MCI & Dementia & p-value \\
\hline $\mathrm{N}$ & 24 & 58 & 14 & - \\
\% Female & 45.8 & 56.9 & 71.4 & 0.147 \\
\% AD aetiology & 4.2 & 53.4 & 57.1 & $<.001$ \\
\% non-AD aetiology & 12.5 & 12.1 & 21.4 & 0.292 \\
\% Unknown aetiology & 83.3 & 34.5 & 21.4 & $\mathbf{0 . 0 0 1}$ \\
AGE M(SD) & $69.6(8.7)$ & $74.2(6.3)$ & $73.9(6.7)$ & 0.064 \\
YoE M(SD) & $15.7(3.7)$ & $13.4(3.9)$ & $10.4(3.7)$ & $\mathbf{0 . 0 0 1}$ \\
MMSE M(SD) & $28.2(1.7)$ & $25.8(2.6)$ & $18.8(4.8)$ & $<.001$ \\
\hline
\end{tabular}

Legend: $\mathrm{CU}=$ cognitively unimpaired; $\mathrm{MCI}=$ mild cognitive impairment; $\mathrm{YoE}=$ years of education; MMSE = Mini-Mental State Examination; $\mathrm{M}(\mathrm{SD})=$ mean (standard deviation); -value was estimated using Chi-squared test for gender and aetiology, and Kruskall-Wallis test for AGE, YoE and MMSE; Etiology was determined by a neurologist on the basis of biomarkers and clinical assessment.

\subsection{Image acquisition parameters}

Structural and functional data were acquired using a 3T Siemens Magnetom Skyra utilising a 64 phased array head coil, at the radiology and neuroradiology division, Geneva University Hospitals, Geneva, Switzerland. An EPI-BOLD sequence was used to collect functional data from 35 interleaved slices (slice thickness $=3 \mathrm{~mm}$; multi-slice mode $=$ interleaved; FoV $=192 \times 192 \times 105 \mathrm{~mm}$; voxel size $=$ $3 \mathrm{~mm}$ isotropic; $\mathrm{TR}=2000 \mathrm{~ms}, \mathrm{TE}=30 \mathrm{~ms}$; flip-angle $=90^{\circ}$; GRAPPA acceleration factor $=2$, timepoints $=200$ ). Whole-brain T1-weighted anatomical images were acquired using a 3D MPRAGE sequence (slice thickness $=0.9 \mathrm{~mm} ; \mathrm{FoV}=263 \times 350 \times 350 \mathrm{~mm}$; voxel size $=1 \mathrm{~mm}$ isotropic; $\mathrm{TR}=$ $1930 \mathrm{~ms} ; \mathrm{TE}=2.36 \mathrm{~ms}$, flip-angle $=8^{\circ}$; GRAPPA acceleration factor $=3$ ).

\subsection{Image processing}

fMRI data were preprocessed using in-house MATLAB code based on state-of-the-art fMRI processing guidelines ${ }^{44,75,79}$. Below follows a brief description of these steps.

Structural images were first denoised to improve the signal-to-noise ratio ${ }^{80}$, bias-field corrected, and then segmented (FSL FAST) to extract white matter, grey matter and cerebrospinal fluid (CSF) tissue masks. These masks were warped in each individual subject's functional space by means of subsequent linear and non-linear registrations (FSL flirt 6dof, FSL flirt 12dof and FSL fnirt). BOLD volume unwarping with applytopup, slice timing correction (slicetimer), realignment (mcflirt), normalisation to mode 1000, demeaning and linear detrending (MATLAB detrend), 
regression (MATLAB regress) of 18 signals: 3 translations, 3 rotations, and 3 tissue-based regressors (mean signal of whole-brain, white matter (WM) and cerebrospinal fluid (CSF), as well as 9 corresponding derivatives (backwards difference; MATLAB). A scrubbing procedure censoring high head motion volumes was based on two metrics: frame displacement (FD, in mm), and DVARS (D referring to temporal derivative of BOLD time courses, VARS referring to root mean square variance over voxels) from ${ }^{44}$. Specifically, we used the standardised DVARS as proposed in ${ }^{81}$. We also used SD (standard deviation of the BOLD signal within brain voxels at every time-point). The FD and DVARS vectors (obtained with fsl_motion_outliers) were used to tag outlier BOLD volumes with FD $>0.3 \mathrm{~mm}$ and standardised DVARS $>1.7$. The SD vector obtained with Matlab was used to tag outlier BOLD volumes higher than the $75^{\text {th }}$ percentile +1.5 of the interquartile range per FSL recommendation ${ }^{82}$. Note that there was no significant difference neither in the percentage of censored volumes nor in the average FD between the groups ( $p>.513)$ and/or test-retest scans ( $>$ >.680).

A bandpass first-order Butterworth filter $[0.01 \mathrm{~Hz}, 0.15 \mathrm{~Hz}]$ was applied to all BOLD timeseries at the voxel level (MATLAB butter and filtfilt). The first three principal components of the BOLD signal in the WM and CSF tissue were regressed out of the gray matter (GM) signal (MATLAB, pca and regress) at the voxel level. A whole-brain data-driven functional parcellation based on 278 regions, as obtained by ${ }^{83}$, was projected into each subject's T1 space (FSL flirt 6dof, FSL flirt 12dof and finally FSL fnirt) and then into the native EPI space of each subject. We also applied FSL boundary-based-registration ${ }^{84}$ to improve the registration of the structural masks and the parcellation to the functional volumes.

Due to the top-to-bottom extension of the FoV, BOLD signal from some ROIs was missing in some cases. When signal from an ROI was not available in more than $25 \%$ of subjects it was excluded from the analyses for all (i.e. for a total of $25 \mathrm{ROIs}$, corresponding to $67 \%$ of the cerebellum ROIs and $16 \%$ of ROIs from subcortical regions); when signal was absent in less than $25 \%$ of subjects (this concerned a total of 44 ROIs) FC value was replaced in the missing cases with the average FC of the intact ROIs in the rest of the sample. This allowed to a) maintain constant across subjects the number of ROIs included in the analyses, b) avoid to drop data in the entire sample when signal was not available only for a few cases, c) avoid to introduce bias driven by ROIs with no value.

We estimated individual FC matrices using Pearson's correlation coefficient between the averaged signals of all region pairs. The resulting individual FC matrices were composed of 278 cortical and subcortical nodes. Finally, the resulting functional connectomes were ordered according to seven cortical resting state networks (RSNs) as proposed by ${ }^{85}$, plus one additional network including subcortical regions (similarly to ${ }^{86}$, see also Fig. 1B).

\subsection{Functional Connectivity and Fingerprinting}

We first assessed the reliability or fingerprinting of FC. At the whole-brain level, fingerprint was calculated for each subject(s) as test-retest similarity between the FCs computed across the first 
100 and second 100 volumes within the same scan (ISelf, Fig 1C). Let us note that $\mathrm{N}$ is the total number of subjects in each group, and we denote the set of subjects as $S u b j$.

$$
\operatorname{ISelf}(s)=\operatorname{corr}\left(F C_{\text {test }}(s), F C_{\text {retest }}(s)\right)
$$

Then, for each subject(s) we computed an index of the FCs similarity with the other subjects(i) in their group (IOthers), as follows:

$$
\operatorname{IOthers}(s)=\frac{\sum_{i \neq s}\left(\operatorname{corr}\left(F C_{\text {test }}(s), F C_{\text {retest }}(i)\right)+\operatorname{corr}\left(F C_{\text {retest }}(s), F C_{\text {test }}(i)\right)\right)}{2 N-2}
$$

A second metric, IDiff (Fig. 1B), provides a group-level estimate of the within- (ISelf) and between-subjects (IOthers) test-retest reliability distance:

$$
\text { IDiff }=\operatorname{mean}_{s \in \text { Subj }} \operatorname{ISelf}(s)-\text { mean }_{s \in \text { Subj }} \operatorname{IOthers}(s)
$$

Finally, we measured the Success-rate ${ }^{7}$ of the identification procedure as percentage of cases with higher within- (ISelf) vs. between-subjects (IOthers) test-retest reliability.

These metrics have been introduced and estimated in healthy populations in previous work ${ }^{12}$. We performed a permutation testing analysis to compare Success-rate and IDiff from 1000 surrogate datasets of random ID matrices against the real value ${ }^{48}$.

Spatial specificity of FC fingerprints was derived using edgewise intra-class correlation (ICC) for each group. ICC coefficients quantify the degree of similarity between observations/measures and find high applicability in reliability studies ${ }^{87}$. The higher the ICC coefficient, the stronger the agreement between two observations. Here, ICC quantifies the similarity between test and retest for each edge (FC between 2 regions), such that the higher the ICC of an edge, the more that edge's connectivity is similar across test and retest or, in other words, the higher the 'fingerprint' of that edge. In order to control for sample size differences across groups, bootstrapping was used to accurately estimate edgewise fingerprints: for each group, ICC was calculated across test and retest for subsets of randomly chosen $\mathrm{N}=10$ subjects, across 100 bootstrap runs, and then averaged within each group.

\subsection{Dynamic Functional Connectivity and connectome flexibility}

In addition to the exploration of FC fingerprints across brain ageing, we also assessed the functional flexibility of individual FCs across time using sliding window analyses ${ }^{88}$. In the sliding window approach, the input data is the time course of regional BOLD activity and FC is computed as pairwise Pearson correlation across ROIs as in static FC, yet this time over a temporal window of a shorter chosen length ( $\mathrm{W}_{\text {length }}$, i.e., from time $\mathrm{t}=1$ to time $\mathrm{t}=\mathrm{W}$ ). Then, the window is shifted by a step 
$\mathrm{T}[\mathrm{t}=1+\mathrm{T}, \mathrm{t}=\mathrm{W}+\mathrm{T}]$ and the calculations are reiterated over a number of windows, depending on the length of the entire acquisition protocol. This process results in a set of connectivity matrices (i.e., the dynamic FC, dFC Fig. 1D).

We calculated dFC across 278 brain regions ${ }^{83}$ with window $\mathrm{W}_{\text {length }}=30$ volumes and step $\mathrm{T}=5$ volumes, for a total of $\mathrm{N}=35$ windows, each lasting $6 \mathrm{~s}\left[\mathrm{~W}_{\text {length }} * \mathrm{TR}=30 * 2000 \mathrm{~ms}\right.$ ]. For each subject, we defined edge-wise (i,j) connectome flexibility index as the standard deviation $(\sigma)$ of the specific FC edge over $\mathrm{N}=35$ windows:

$$
\text { Flexibility }_{(i, j)}(s)=\sigma_{W}\left(F C_{(i, j, W)}(s)\right)
$$

This resulted in a $278 \times 278$ "flexibility matrix" for each subject, revealing the individuals' flexibility of functional connections across regions over time. Edge-wise dFC Flexibility was averaged across groups using bootstrapping to control for sample size differences, i.e., average was calculated for $\mathrm{N}=100$ times for subsets of randomly chosen $\mathrm{N}=10$ subjects for each group and then averaged.

\subsection{Grey matter volume quantification}

For each subject, grey matter volume was quantified from T1 weighted images as the number of voxels within each parcel of Shen parcellation, and then normalised by the total intracranial volume. Group-wise, structural covariance matrices were derived as across-subject Spearman correlation of grey matter volume, yielding a $278 \times 278$ covariance matrix, where positive correlations indicate a similar grey matter volume trend within a given ROI pair ${ }^{89}$.

\section{Acknowledgements}

SS was supported by a grant from the Swiss National Science Foundation [SNSF 320030_185028, PI: VG]. Data acquisition was granted by SNSF 320030_185028 and 169876 (PI: VG) and Fonds Startup du département de radiologie et informatique médicale, Université de Genève, Faculté de Médecine (PI: SS). Data for this study were collected at the Centre de la mémoire, Geneva University and University Hospitals, thanks to funds from: Association Suisse pour la Recherche sur l'Alzheimer, Genève; Fondation Segré, Genève; Ivan Pictet, Genève; Fondazione Agusta, Lugano; Fondation Chmielewski, Genève; Velux Stiftung; Swiss National Science Foundation (projects n.320030_182772); Horizon 2020 (projects n. 667375); Human Brain Project; Innovative Medicines Initiatives (IMI contract n. 115736 and 115952). EA acknowledges financial support from the SNSF Ambizione project "Fingerprinting the brain: Network science to extract features of cognition, behaviour and dysfunction" (grant number PZ00P2_185716). VG is supported also from the Velux Foundation, the Aetas Foundation, the Schmidheiny Foundation and research/teaching support 
through her institution from Siemens Healthineers, GE Healthcare, Roche, Merck, Cerveau Technologies and Life Molecular Imaging.

We would like to thank Dr. Benedetta Franceschiello for the insightful discussions, and the assistance with coding, mathematics and statistics. We thank Emahnuel Troisi-Lopez and Pierpaolo Sorrentino for the insightful comments. We are indebted to the patients, their carers, and the volunteers for taking part in this study, and with the staff of the Geneva Memory Centre for data collection.

\section{Authors contributions}

SS, EA and VG conceptualised the study. EA and SS processed the (f)MRI data and wrote the code for the analyses. SS performed the static and dynamic functional connectivity analyses, and fingerprinting analyses. SS, EA and VG interpreted the results. SS and EA wrote the first draft of the manuscript; SS, EA and VG reviewed and edited. GBF, FR and ST provided the data, assisted with the database and the clinical assessment. MS and KOL acquired the f(MRI) data. SA assisted with (f)MRI data processing and figures. All co-authors approved the final draft of the manuscript.

\section{Competing interests}

All authors declare no competing interests.

\section{$\underline{\text { References }}$}

1. Van Essen, D. C. et al. The Human Connectome Project: A data acquisition perspective. NeuroImage 62, 2222-2231 (2012).

2. Van Essen, D. C. et al. The WU-Minn Human Connectome Project: An overview. NeuroImage 80, 62-79 (2013).

3. Weiner, M. W. et al. The Alzheimer's Disease Neuroimaging Initiative 3: Continued innovation for clinical trial improvement. Alzheimer's \&amp; Dementia 13, 561-571 (2017).

4. Bullmore, E. \& Sporns, O. Complex brain networks: graph theoretical analysis of structural and functional systems. Nat Rev Neurosci 10, 186-198 (2009).

5. Sporns, O. The human connectome: a complex network: The human connectome. Annals of the New York Academy of Sciences 1224, 109-125 (2011).

6. Fornito, A., Zalesky, A. \& Bullmore, E. Fundamentals of Brain Network Analyses. (Academic 
Press, 2016).

7. Finn, E. S. et al. Functional connectome fingerprinting: identifying individuals using patterns of brain connectivity. Nat Neurosci 18, 1664-1671 (2015).

8. Miranda-Dominguez, O. et al. Connectotyping: Model Based Fingerprinting of the Functional Connectome. PLoS ONE 9, e111048 (2014).

9. Finn, E. S. \& Rosenberg, M. D. Beyond fingerprinting: Choosing predictive connectomes over reliable connectomes. NeuroImage 239, 118254 (2021).

10. Rosenberg, M. D. et al. A neuromarker of sustained attention from whole-brain functional connectivity. Nat Neurosci 19, 165-171 (2016).

11. Abbas, K. et al. GEFF: Graph embedding for functional fingerprinting. NeuroImage 221, 117181 (2020).

12. Amico, E. \& Goñi, J. The quest for identifiability in human functional connectomes. Sci Rep $\mathbf{8}$, 8254 (2018).

13. Bari, S., Amico, E., Vike, N., Talavage, T. M. \& Goñi, J. Uncovering multi-site identifiability based on resting-state functional connectomes. NeuroImage 202, 115967 (2019).

14. Jirsa, V. K. et al. The Virtual Epileptic Patient: Individualized whole-brain models of epilepsy spread. NeuroImage 145, 377-388 (2017).

15. Liu, W., Kohn, N. \& Fernández, G. Intersubject similarity of personality is associated with intersubject similarity of brain connectivity patterns. NeuroImage 186, 56-69 (2019).

16. Shen, X. et al. Using connectome-based predictive modeling to predict individual behavior from brain connectivity. Nat Protoc 12, 506-518 (2017).

17. Sui, J., Jiang, R., Bustillo, J. \& Calhoun, V. Neuroimaging-based Individualized Prediction of Cognition and Behavior for Mental Disorders and Health: Methods and Promises. Biological Psychiatry 88, 818-828 (2020).

18. Sorrentino, P. et al. Clinical connectome fingerprints of cognitive decline. NeuroImage $\mathbf{2 3 8}$, $118253(2021)$.

19. Svaldi, D. O. et al. Optimizing differential identifiability improves connectome predictive modeling of cognitive deficits from functional connectivity in Alzheimer's disease. Hum Brain 
Mapp 42, 3500-3516 (2021).

20. Palop, J. J., Chin, J. \& Mucke, L. A network dysfunction perspective on neurodegenerative diseases. Nature 443, 768-773 (2006).

21. Delbeuck, X. Alzheimer's Disease as a Disconnection Syndrome? 14 (2003).

22. Badhwar, A. et al. Resting-state network dysfunction in Alzheimer's disease: A systematic review and meta-analysis. Alzheimer's \& Dementia 8, 73-85 (2017).

23. Pievani, M., Filippini, N., van den Heuvel, M. P., Cappa, S. F. \& Frisoni, G. B. Brain connectivity in neurodegenerative diseases - from phenotype to proteinopathy. Nat Rev Neurol 10, 620-633 (2014).

24. Yu, M., Sporns, O. \& Saykin, A. J. The human connectome in Alzheimer disease - relationship to biomarkers and genetics. Nat Rev Neurol (2021) doi:10.1038/s41582-021-00529-1.

25. de Haan, W. et al. Disrupted modular brain dynamics reflect cognitive dysfunction in Alzheimer's disease. NeuroImage 59, 3085-3093 (2012).

26. Yu, M. et al. Different functional connectivity and network topology in behavioral variant of frontotemporal dementia and Alzheimer's disease: an EEG study. Neurobiology of Aging 42, $150-162(2016)$.

27. Agosta, F. et al. Resting state fMRI in Alzheimer's disease: beyond the default mode network. Neurobiology of Aging 33, 1564-1578 (2012).

28. Stam, C. J. Modern network science of neurological disorders. Nat Rev Neurosci 15, 683-695 (2014).

29. Tijms, B. M. et al. Alzheimer's disease: connecting findings from graph theoretical studies of brain networks. Neurobiology of Aging 34, 2023-2036 (2013).

30. Wang, K. et al. Altered functional connectivity in early Alzheimer's disease: A resting-state fMRI study. Hum. Brain Mapp. 28, 967-978 (2007).

31. Brier, M. R. et al. Loss of Intranetwork and Internetwork Resting State Functional Connections with Alzheimer's Disease Progression. Journal of Neuroscience 32, 8890-8899 (2012).

32. Damoiseaux, J. S. Resting-state fMRI as a biomarker for Alzheimer's disease? Alzheimers Res Ther 4, 8 (2012). 
33. Jack, C. R. et al. Amyloid-first and neurodegeneration-first profiles characterize incident amyloid PET positivity. Neurology 81, 1732-1740 (2013).

34. Schultz, A. P. et al. Phases of Hyperconnectivity and Hypoconnectivity in the Default Mode and Salience Networks Track with Amyloid and Tau in Clinically Normal Individuals. J. Neurosci. 37, 4323-4331 (2017).

35. Buckner, R. L. et al. Cortical Hubs Revealed by Intrinsic Functional Connectivity: Mapping, Assessment of Stability, and Relation to Alzheimer's Disease. Journal of Neuroscience 29, 18601873 (2009).

36. Crossley, N. A. et al. The hubs of the human connectome are generally implicated in the anatomy of brain disorders. Brain 137, 2382-2395 (2014).

37. van den Heuvel, M. P. \& Sporns, O. Network hubs in the human brain. Trends in Cognitive Sciences 17, 683-696 (2013).

38. Palmqvist, S. et al. Earliest accumulation of $\beta$-amyloid occurs within the default-mode network and concurrently affects brain connectivity. Nat Commun 8, 1214 (2017).

39. Yu, M. et al. Selective impairment of hippocampus and posterior hub areas in Alzheimer's disease: an MEG-based multiplex network study. Brain 140, 1466-1485 (2017).

40. Franzmeier, N. et al. Patient-centered connectivity-based prediction of tau pathology spread in Alzheimer's disease. Sci. $A d v . \mathbf{6},(2020)$.

41. Franzmeier, N. et al. Functional connectivity associated with tau levels in ageing, Alzheimer's, and small vessel disease. Brain 142, 1093-1107 (2019).

42. Franzmeier, N. et al. Functional brain architecture is associated with the rate of tau accumulation in Alzheimer's disease. Nat Commun 11, 347 (2020).

43. Jack, C. R. et al. NIA-AA Research Framework: Toward a biological definition of Alzheimer's disease. Alzheimer's \& Dementia 14, 535-562 (2018).

44. Power, J. D. et al. Methods to detect, characterize, and remove motion artifact in resting state fMRI. NeuroImage 84, 320-341 (2014).

45. Chiesa, P. A., Cavedo, E., Lista, S., Thompson, P. M. \& Hampel, H. Revolution of Resting-State Functional Neuroimaging Genetics in Alzheimer's Disease. Trends in Neurosciences 40, 469-480 
(2017).

46. Shaw, L. M., Korecka, M., Clark, C. M., Lee, V. M.-Y. \& Trojanowski, J. Q. Biomarkers of neurodegeneration for diagnosis and monitoring therapeutics. Nat Rev Drug Discov 6, 295-303 (2007).

47. Van De Ville, D., Farouj, Y., Preti, M. G., Liégeois, R. \& Amico, E. When makes you unique: temporality of the human brain fingerprint. http://biorxiv.org/lookup/doi/10.1101/2021.03.24.436733 (2021) doi:10.1101/2021.03.24.436733.

48. Sareen, E. et al. Exploring MEG brain fingerprints: Evaluation, pitfalls, and interpretations. NeuroImage 240, 118331 (2021).

49. McGraw, K. O. \& Wong, S. P. Forming Inferences About Some Intraclass Correlation Coefficients. Psychological Methods 17 (1996) doi:1082-989X/96/S3.00.

50. Shrout, P. E. \& Fleiss, J. L. Intraclass Correlations: Uses in Assessing Rater Reliability. Psychological Bulletin 86, 420-428 (1979).

51. Albert, M. S. et al. The diagnosis of mild cognitive impairment due to Alzheimer's disease: Recommendations from the National Institute on Aging-Alzheimer's Association workgroups on diagnostic guidelines for Alzheimer's disease. Alzheimer's \& Dementia 7, 270-279 (2011).

52. Frisoni, G. B. et al. The probabilistic model of Alzheimer disease: the amyloid hypothesis revised. Nat Rev Neurosci (2021) doi:10.1038/s41583-021-00533-w.

53. Jackson, R. L. The neural correlates of semantic control revisited. NeuroImage 224, 117444 (2021).

54. Jefferies, E. The neural basis of semantic cognition: Converging evidence from neuropsychology, neuroimaging and TMS. Cortex 49, 611-625 (2013).

55. Jefferies, E. \& Lambon Ralph, M. A. Semantic impairment in stroke aphasia versus semantic dementia: a case-series comparison. Brain 129, 2132-2147 (2006).

56. Lambon-Ralph, M. A., Jefferies, E., Patterson, K. \& Rogers, T. T. The neural and computational bases of semantic cognition. Nat Rev Neurosci 18, 42-55 (2017).

57. Badre, D., Poldrack, R. A., Paré-Blagoev, E. J., Insler, R. Z. \& Wagner, A. D. Dissociable Controlled Retrieval and Generalized Selection Mechanisms in Ventrolateral Prefrontal Cortex. 
Neuron 47, 907-918 (2005).

58. Barredo, J., Öztekin, I. \& Badre, D. Ventral Fronto-Temporal Pathway Supporting Cognitive Control of Episodic Memory Retrieval. Cerebral Cortex 25, 1004-1019 (2015).

59. Stampacchia, S. et al. Control the source: Source memory for semantic, spatial and self-related items in patients with LIFG lesions. Cortex 119, 165-183 (2019).

60. Stampacchia, S. et al. Shared processes resolve competition within and between episodic and semantic memory: Evidence from patients with LIFG lesions. Cortex 108, 127-143 (2018).

61. Diveica, V., Koldewyn, K. \& Binney, R. J. Establishing a role of the semantic control network in social cognitive processing: A meta-analysis of functional neuroimaging studies. NeuroImage 245, $118702(2021)$.

62. Smallwood, J. et al. The default mode network in cognition: a topographical perspective. Nat Rev Neurosci 22, 503-513 (2021).

63. Bonnici, H. M., Richter, F. R., Yazar, Y. \& Simons, J. S. Multimodal Feature Integration in the Angular Gyrus during Episodic and Semantic Retrieval. Journal of Neuroscience 36, 5462-5471 (2016).

64. Lanzoni, L. et al. The role of default mode network in semantic cue integration. NeuroImage 219, $117019(2020)$.

65. Humphreys, G. F. \& Lambon Ralph, M. A. Mapping Domain-Selective and Counterpointed Domain-General Higher Cognitive Functions in the Lateral Parietal Cortex: Evidence from fMRI Comparisons of Difficulty-Varying Semantic Versus Visuo-Spatial Tasks, and Functional Connectivity Analyses. Cerebral Cortex 27, 4199-4212 (2017).

66. Seghier, M. L. The Angular Gyrus: Multiple Functions and Multiple Subdivisions. Neuroscientist 19, 43-61 (2013).

67. Bagattini, C. et al. Functional Imaging to Guide Network-Based TMS Treatments: Toward a Tailored Medicine Approach in Alzheimer's Disease. Front. Neurosci. 15, 687493 (2021).

68. Pievani, M., Pini, L., Cappa, S. F. \& Frisoni, G. B. Brain networks stimulation in dementia: insights from functional imaging. Current Opinion in Neurology 29, 756-762 (2016).

69. Binney, R. J., Embleton, K. V., Jefferies, E., Parker, G. J. M. \& Lambon Ralph, M. A. The 
Ventral and Inferolateral Aspects of the Anterior Temporal Lobe Are Crucial in Semantic Memory: Evidence from a Novel Direct Comparison of Distortion-Corrected fMRI, rTMS, and Semantic Dementia. Cerebral Cortex 20, 2728-2738 (2010).

70. Rice, G. E., Caswell, H., Moore, P., Hoffman, P. \& Lambon Ralph, M. A. The Roles of Left Versus Right Anterior Temporal Lobes in Semantic Memory: A Neuropsychological Comparison of Postsurgical Temporal Lobe Epilepsy Patients. Cerebral Cortex 28, 1487-1501 (2018).

71. Jefferies, E., Thompson, H., Cornelissen, P. \& Smallwood, J. The neurocognitive basis of knowledge about object identity and events: dissociations reflect opposing effects of semantic coherence and control. Phil. Trans. R. Soc. B 375, 20190300 (2020).

72. Suvà, D. et al. Primary Motor Cortex Involvement in Alzheimer Disease: Journal of Neuropathology and Experimental Neurology 58, 1125-1134 (1999).

73. Vidoni, E. D., Thomas, G. P., Honea, R. A., Loskutova, N. \& Burns, J. M. Evidence of Altered Corticomotor System Connectivity in Early-Stage Alzheimer's Disease. Journal of Neurologic Physical Therapy 36, 8-16 (2012).

74. Birn, R. M. The role of physiological noise in resting-state functional connectivity. NeuroImage 62, 864-870 (2012).

75. Power, J. D., Barnes, K. A., Snyder, A. Z., Schlaggar, B. L. \& Petersen, S. E. Spurious but systematic correlations in functional connectivity MRI networks arise from subject motion. NeuroImage 59, 2142-2154 (2012).

76. Ribaldi, F. et al. Brain connectivity and metacognition in persons with subjective cognitive decline (COSCODE): rationale and study design. Alz Res Therapy 13, 105 (2021).

77. McKhann, G. M. et al. The diagnosis of dementia due to Alzheimer's disease: Recommendations from the National Institute on Aging-Alzheimer's Association workgroups on diagnostic guidelines for Alzheimer's disease. Alzheimer's \&amp; Dementia 7, 263-269 (2011).

78. Le Duff, F. et al. The 2008-2012 French Alzheimer Plan: Description of the National Alzheimer Information System. 13 (2012).

79. Amico, E. et al. Mapping the functional connectome traits of levels of consciousness. NeuroImage 148, 201-211 (2017). 
80. Coupé, P., Manjón, J. V., Robles, M. \& Collins, D. L. Adaptive multiresolution non-local means filter for three-dimensional magnetic resonance image denoising. IET Image Process. 6, 558 (2012).

81. Afyouni, S. \& Nichols, T. E. Insight and inference for DVARS. NeuroImage 172, 291-312 (2018).

82. Jenkinson, M., Bannister, P., Brady, M. \& Smith, S. Improved Optimization for the Robust and Accurate Linear Registration and Motion Correction of Brain Images. NeuroImage 17, 825-841 (2002).

83. Shen, X., Tokoglu, F., Papademetris, X. \& Constable, R. T. Groupwise whole-brain parcellation from resting-state fMRI data for network node identification. NeuroImage 82, 403-415 (2013).

84. Greve, D. N. \& Fischl, B. Accurate and robust brain image alignment using boundary-based registration. NeuroImage 48, 63-72 (2009).

85. Yeo, B. T. T. et al. The organization of the human cerebral cortex estimated by intrinsic functional connectivity. $J$ Neurophysiol 106, 41 (2011).

86. Amico, E. et al. The disengaging brain: Dynamic transitions from cognitive engagement and alcoholism risk. NeuroImage 209, 116515 (2020).

87. Koch, G. G. Intraclass Correlation Coefficient. Wiley StatsRef: Statistics Reference Online 7 (2014) doi:10.1002/9781118445112.stat02461.

88. Preti, M. G., Bolton, T. A. \& Van De Ville, D. The dynamic functional connectome: State-of-theart and perspectives. NeuroImage 160, 41-54 (2017).

89. Di, X. et al. Do all roads lead to Rome? A comparison of brain networks derived from intersubject volumetric and metabolic covariance and moment-to-moment hemodynamic correlations in old individuals. Brain Struct Funct 222, 3833-3845 (2017). 
bioRxiv preprint doi: https://doi.org/10.1101/2022.02.04.479112; this version posted February 8, 2022. The copyright holder for this preprint (which was not certified by peer review) is the author/funder, who has granted bioRxiv a license to display the preprint in perpetuity. It is made available under aCC-BY-NC-ND 4.0 International license. 